\title{
YiQiFuMai powder injection ameliorates blood- brain barrier dysfunction and brain edema after focal cerebral ischemia-reperfusion injury in mice
}

This article was published in the following Dove Press journal:

Drug Design, Development and Therapy

14 January 2016

Number of times this article has been viewed

\author{
Guosheng Cao \\ Xinyi Ye \\ Yingqiong $\mathrm{Xu}$ \\ Mingzhu Yin \\ Honglin Chen \\ Junping Kou \\ Boyang Yu
}

Jiangsu Key Laboratory of TCM

Evaluation and Translational Research, Department of Complex Prescription of TCM, China Pharmaceutical University, Nanjing, People's Republic of China
Correspondence: Junping Kou Jiangsu Key Laboratory of TCM Evaluation and Translational Research, Department of Complex Prescription of TCM, China Pharmaceutical University, 639 Longmian Road, Nanjing 211198 , People's Republic of China Tel/fax +862586185158

Email junpingkou@cpu.edu.cn
Abstract: YiQiFuMai powder injection (YQFM) is a modern preparation derived from the traditional Chinese medicine Sheng-Mai-San. YQFM is widely used in clinical practice in the People's Republic of China, mainly for the treatment of microcirculatory disturbance-related diseases. However, little is known about its role in animals with ischemic stroke. The aim of this study was to examine the effect of YQFM on brain edema and blood-brain barrier (BBB) dysfunction induced by cerebral ischemia-reperfusion (I/R) injury. Male C57BL/6J mice underwent right middle cerebral artery occlusion for 1 hour with a subsequent 24-hour reperfusion to produce I/R injury. YQFM (three doses: 0.336, 0.671, and $1.342 \mathrm{~g} / \mathrm{kg}$ ) was then given intraperitoneally (IP). The results demonstrated that YQFM significantly decreased infarct size, improved neurological deficits, reduced brain water content, and increased cerebral blood flow after I/R injury. ${ }^{18} \mathrm{~F}$-fluorodeoxyglucose micro-positron emission tomography imaging and hematoxylin and eosin staining results indicated that YQFM is able to ameliorate brain metabolism and histopathological damage after I/R. Moreover, YQFM administration reduced BBB leakage and upregulated the expression of zona occludens-1 (ZO-1) and occludin, which was confirmed by Evans Blue extravasation, Western blotting, and immunofluorescence assay. Our findings suggest that YQFM provides protection against focal cerebral I/R injury in mice, possibly by improving BBB dysfunction via upregulation of the expression of tight junction proteins.

Keywords: YiQiFuMai powder injection, YQFM, ischemic stroke, blood-brain barrier, microvascular permeability, tight junctions

\section{Introduction}

Ischemic stroke is a destructive cerebrovascular disease and a leading cause of death worldwide. ${ }^{1}$ Currently, recombinant tissue plasminogen activator is the only therapeutic agent available for the treatment of ischemic stroke. However, $<5 \%$ of patients with stroke recover, owing to the narrow therapeutic window, ${ }^{2,3}$ risk of intracerebral hemorrhage, and multiple contraindications. ${ }^{4}$ Given the devastating impact and social burden of this damaging cerebrovascular event, the development of optimum treatments for ischemic stroke is urgently needed. ${ }^{5}$

Abundant evidence has confirmed that the disruption of the blood-brain barrier (BBB) and the consequent brain edema are major contributors to the pathogenesis of ischemic stroke. Intravascular proteins and fluid penetrate into the cerebral parenchymal extracellular space, leading to vasogenic cerebral edema and reduced blood flow to neurons, resulting in an irreversible apoptosis. ${ }^{6,7}$ The BBB is not a rigid structure but a dynamic interface, with a range of interrelated functions that result from effective tight junctions (TJs), trans-endothelial transport systems, enzymes, and the regulation of leukocyte permeation. TJs are the most apical structure within the intercellular cleft, 
limiting the paracellular flux of hydrophilic molecules across the BBB. TJs consist of several types of integral transmembrane and cytoplasmic accessory proteins, of which occludin and zona occludens-1 (ZO-1) play key roles in BBB dysfunction after ischemic stroke. ${ }^{8,9}$ Thus, protecting the BBB may be a promising strategy for developing new clinical therapies for ischemic stroke. ${ }^{10}$

A number of studies have indicated that traditional Chinese medicine preparations, such as XueShuanTong injection (XST), ${ }^{11}$ Danhong injection, ${ }^{12}$ and Buyanghuanwu decoction, ${ }^{13}$ have reliable curative efficacy in the prevention and treatment of stroke. YiQiFuMai powder injection (YQFM), a modern preparation based on a well-known complex prescription, Sheng-Mai-San, was approved in 2007 by the China Food and Drug Administration for the treatment of microcirculatory disturbance-related diseases in the People's Republic of China. ${ }^{14,15}$ YQFM is composed of Panax ginseng CA Mey., Ophiopogon japonicus (Thunb.) Ker-Gawl, and Schisandra chinensis (Turcz.) Baill (1:3:1.5). ${ }^{16}$ The bioactive compounds and their chemical structures were identified by tandem mass spectrophotometry in negative electrospray ionization mode. ${ }^{17}$ Considerable numbers of previous studies have indicated that YQFM is widely used for the treatment of cardiovascular disease. ${ }^{17,18}$ We previously predicted the multitarget mechanism of YQFM in cardiocerebral ischemic disease, based on network pharmacology. ${ }^{19}$ However, its related pharmacological activity and potential mechanism in ischemic stroke in animals remain unclear. Therefore, in the current study, we investigated the effects of YQFM on brain edema and BBB dysfunction induced by cerebral ischemia-reperfusion (I/R) compared with XST and explored the possible modulation of TJs in $\mathrm{I} / \mathrm{R}$ induced by middle cerebral artery occlusion (MCAO) in mice. Our findings provide some evidence for the clinical application of YQFM in ischemic stroke.

\section{Materials and methods Reagents}

YQFM (batch number 20121210) was purchased from Tasly Pharmaceutical Co., Ltd. (Tianjin, People's Republic of China). An ultrahigh performance liquid chromatography (UHPLC) fingerprint (Figure 1) was used to control the quality of YQFM in the current study. Based on this fingerprint, we established an optimum and easily controlled method for the analysis of YQFM in our laboratory. ${ }^{18}$ XST was purchased from Livzon Pharmaceutical Group Inc. (Guangdong, People's Republic of China). Fluorescein-isothiocyanate-conjugated goat antirabbit immunoglobulin $\mathrm{G}$ and horseradish-peroxidase-conjugated goat antirabbit and anti-mouse immunoglobulin $\mathrm{G}$ were purchased from Jackson ImmunoResearch Laboratories, Inc. (West Grove, PA, USA). All chemicals were of the commercially available highest purity.

\section{UHPLC analysis}

The injection of YQFM was prepared as reported previously. ${ }^{13}$ A UHPLC system (Acquity ${ }^{\mathrm{TM}}$ UPLC; Waters Corporation, Milford, MA, USA), equipped with a diode-array detection (DAD) system and a Zorbax Eclipse plus $\mathrm{C}_{18}$ column $(50 \mathrm{~mm} \times 2.1 \mathrm{~mm} \times 1.8 \mu \mathrm{m})$, was used for the UHPLC analysis. The monitoring detection was set at $203 \mathrm{~nm}$, and diodearray detection spectra were recorded from 190 to $400 \mathrm{~nm}$. The sample injection volume was $5 \mu \mathrm{L}$, and the mobile phase was water:formic acid (100:0.01, v/v) (A) and acetonitrile (B). The UPLC eluting conditions were as follows: $2 \%-20 \%$ B for 0-3 minutes; $20 \%-30 \%$ B for 3-9 minutes; 30\%-38\% B for 9-12 minutes; 38\%-40.5\% B for 12-14.5 minutes;

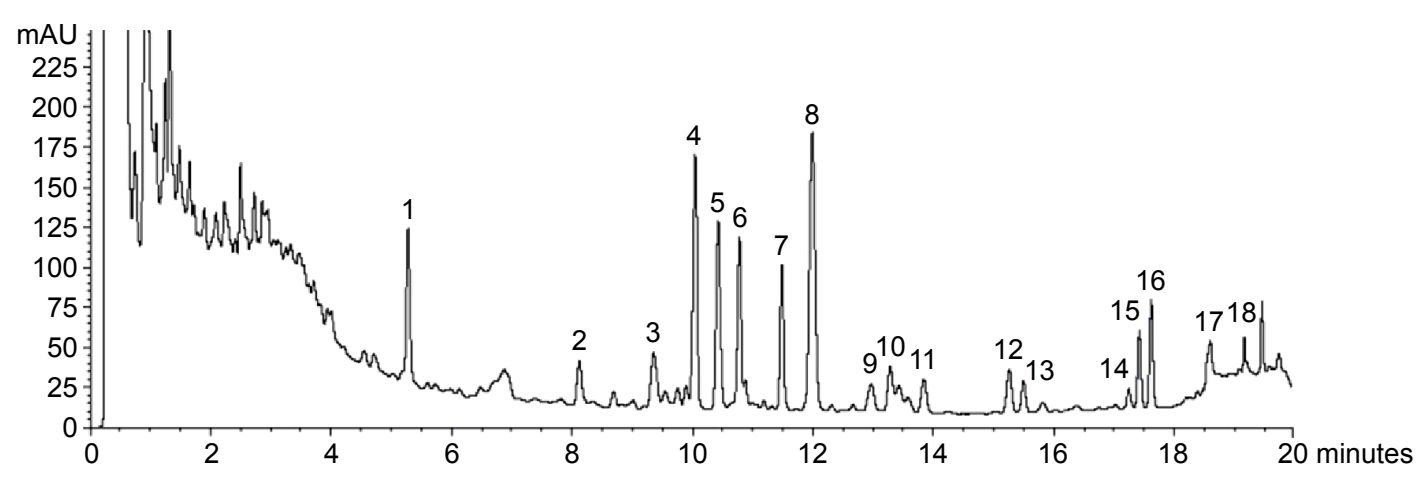

Figure I Chromatogram profile of YQFM.

Notes: Identification of main components in YQFM was as follows: I, ginsenoside RgI; 2, ginsenoside Rf; 3, 20(S)-ginsenoside Rg2; 4, ginsenoside RbI; 5, ginsenoside Rc; 6, ginsenoside Rb2; 7, ginsenoside Rd; 8, schizandrol A; 9, ginsenoside Rg6; 10, ginsenoside F4; II, ginsenoside Rh4/Rk3; I2, 20(S)-ginsenoside Rg3; I3, 20(R)-ginsenoside Rg3; 14, ginsenoside Rs3/isomer; I5, ginsenoside RkI/Rg5; 16, ginsenoside RkI/Rg5; 17, schizandrin A; I8, schizandrin B.

Abbreviation: YQFM, YiQiFuMai powder injection. 
40.5\%-55\% B for 14.5-17.5 minutes; 55\%-99\% B for 17.5-19.5 minutes; and 99\%-2\% B for 19.5-20 minutes. The flow rate was $0.5 \mathrm{~mL} / \mathrm{min}$, and the column temperature was set at $25^{\circ} \mathrm{C}$.

\section{Animals}

C57BL/6J mice weighing 18-22 g were provided by the Reference Animal Research Centre of Yangzhou University (Yangzhou, People's Republic of China; certificate no SCXK 2014-0004). All procedures and assessments were approved by the Animal Ethics Committee of the School of Chinese Materia Medica, China Pharmaceutical University. These experiments were carried out in accordance with the National Institutes of Health Guide for the Care and use of Laboratory Animals (National Institutes of Health Publication No 80-23, revised in 1996). Before performing the experiments, all animals were randomized into experimental groups, and the indices were measured by operators blinded to the study procedures.

\section{Focal cerebral I/R}

Stroke was induced by MCAO and reperfusion in C57BL/6J mice as reported previously. ${ }^{20}$ Briefly, each mouse was anesthetized with $4 \%$ chloral hydrate $(0.1 \mathrm{~mL}$ per $10 \mathrm{~g}$ body weight $)$ IP, then the neck vessels were exposed by a midline incision, and the branches of the right external carotid artery were carefully isolated and cauterized. A 6-0 nylon monofilament suture, blunted at the tip and coated with $1 \%$ poly-L-lysine, was advanced 9-10 $\mathrm{mm}$ into the internal carotid to occlude the origin of the middle cerebral artery. The temperature of the animal was maintained at $37.0^{\circ} \mathrm{C} \pm 0.5^{\circ} \mathrm{C}$ with a heating pad (ALCBIO, Shanghai, People's Republic of China) during surgery and ischemia. The same treatment was carried out on mice in that sham group except that the suture was not advanced into the internal carotid artery. A laser Doppler flowmeter (FLPI2, Moor Instruments Ltd., Axminster, UK) was used to confirm that middle cerebral artery blood flow had decreased $<30 \%$ of the basic cerebral blood flow (CBF) immediately after the occlusion to. ${ }^{21}$ Animals whose blood flow decreased $<30 \%$ of pre-ischemia levels were used for further study.

\section{Evaluation of infarct volume, neurological deficits, and brain water content}

To confirm whether YQFM exerts protective effects in mice, the animals were randomly divided into six groups ( $n=6$ for each group): sham, I/R, YQFM (dissolved in $0.9 \%$ sodium chloride at three doses of $0.336,0.671$, and $1.342 \mathrm{~g} / \mathrm{kg}$ ), and XST (also dissolved in $0.9 \%$ sodium chloride at a dose of
$40 \mathrm{mg} / \mathrm{kg}$ in accordance with clinical practice). After MCAO for 1 hour, mice were given YQFM or an equal volume of $0.9 \%$ sodium chloride by IP administration. After 24 hours of reperfusion, mice were anesthetized with $4 \%$ choral hydrate IP; after the animals were dead, the brains were removed, dissected, and sliced; and then five slices ( $1.5 \mathrm{~mm}$ thickness) were sectioned and incubated with 1\% 2,3,5-triphenyltetrazolium chloride (TTC; Sigma-Aldrich Co., St Louis, MO, USA) for 30 minutes in the dark and then fixed with 4\% paraformaldehyde (PFA). Lesioned areas that did not stain red with TTC were quantitatively analyzed by Quantity One analysis software (Bio-Rad Laboratories Inc., Hercules, CA, USA). Infarct volume was calculated using slice thickness and the measured areas of the lesion. Data are expressed as a percentage of total hemispheres. ${ }^{22}$ Behavioral assessment was performed 24 hours after reperfusion. Neurological deficits of the experimental animals were graded on an 18-point scale as previously described. ${ }^{23}$ The measurement of neurological deficits consisted of the following tests: spontaneous activity, symmetry of movements, symmetry of forelimbs, climbing, reaction to touch, and response to vibrissae touch. All six individual tests were scored on a four-point scale as 3, 2, 1, or 0 . Final score was obtained by adding the scores recorded for each individual test, with a maximum score of 18 observed in healthy animals. Brain water content ( $\mathrm{n}=6$ for each group) was determined 24 hours after reperfusion, using the wet-dry method as described previously. ${ }^{24}$ The total brain water was calculated as:

$$
\frac{\text { Wet weight }- \text { Dry weight }}{\text { Wet weight }} \times 100 \%
$$

\section{CBF measurement}

$\mathrm{CBF}$ was measured using laser Doppler flowmetry as described in the "Materials and methods" section. ${ }^{20}$ A computer-controlled optical scanner directed a low-power laser beam over the exposed cortex. The scanner head was positioned parallel to the cerebral cortex at a distance of $\sim 20 \mathrm{~cm} .{ }^{25} \mathrm{~A}$ color-coded image indicating specific relative perfusion levels was displayed on a video monitor. The images were acquired at the onset of ischemia and reperfusion for 24 hours ( $\mathrm{n}=6$ for each group).

\section{Hematoxylin and eosin staining}

Histomorphological analysis was measured by hematoxylin and eosin (H\&E) staining. Brains were immediately taken from mice at 24 hours after reperfusion ( $n=3$ for each group) under deep anesthesia with chloral hydrate and, after fixation by immersing in $10 \%$ phosphate-buffered formalin for 24 hours, were processed routinely for paraffin wax embedding. A series 
of adjacent brain sections ( $5 \mu \mathrm{m}$ thick) were cut from the coronal plane of the wax-embedded tissue and were stained with $\mathrm{H} \& \mathrm{E}$ for histomorphological analysis conducted by a pathologist blinded to the treatment groups. ${ }^{26}$

\section{${ }^{18} \mathrm{~F}$-fluorodeoxyglucose-positron emission tomography imaging}

${ }^{18} \mathrm{~F}$-fluorodeoxyglucose-micro-positron emission tomography $\left({ }^{18} \mathrm{~F}-\mathrm{FDG}-\right.$ micro-PET) scans were performed in mice ( $n=3$ for each group) anesthetized at 23 hours after reperfusion with $4 \%$ isoflurane, with anesthesia maintained with $2 \%-2.5 \%$ of isoflurane in $100 \% \mathrm{O}_{2}$. The tail vein was catheterized with a gauge catheter for intravenous administration of the radiotracer (radiochemical purity was $>95 \%$; provided by Mitro Biotech Co. Ltd., Nanjing, People's Republic of China). Animals were placed into a mouse holder compatible with PET acquisition systems (Trans-PET ${ }^{\circledR}$ Bio-Caliburn ${ }^{\circledR}$ 700; X-Z LAB, Inc., San Ramon, CA, USA), and normothermia was maintained by a water-based heating blanket. ${ }^{27}$ Animals underwent two micro-PET scans to assess glucose metabolism of ${ }^{18} \mathrm{~F}-\mathrm{FDG}$ for each time point and condition (before ischemia and 24 hours after I/R).

\section{Evaluation of BBB permeability}

BBB permeability was assessed by the leakage of Evans Blue (EB) stain into the brain following the tail-vein injection. ${ }^{28}$ Two hours before the animals ( $n=6$ for each group) were euthanized, $0.1 \mathrm{~mL}$ per $10 \mathrm{~g}$ body weight of $2 \% \mathrm{~EB}$ (SigmaAldrich) in normal saline was injected into each animal. The animals were then anesthetized and perfused with normal saline. For the quantitative measurement of EB leakage, the ipsilateral hemisphere was removed and homogenized in $1 \mathrm{~mL}$ of trichloroacetic acid, then centrifuged at $12,000 \times \mathrm{g}$ for 20 minutes. EB concentration was quantitatively determined by measuring the absorbance at $620 \mathrm{~nm}$ of the supernatant with a spectrophotometer. The EB content was quantified as micrograms of EB per gram of tissue, using a standard curve. ${ }^{29}$

\section{Western blotting}

Western blotting ( $\mathrm{n}=3$ for each group) was performed as described previously. ${ }^{25,28}$ Proteins extracted from the cortex of the ischemic side in the operated mice, and the corresponding areas of sham-operated mice were used for Western blot analysis. Membranes were incubated overnight at $4^{\circ} \mathrm{C}$ with the appropriate primary antibodies: ZO-1 (1:100; ab59720), occludin (1:200; ab31721) (both were purchased from Abcam, Cambridge, UK), and glyceraldehyde 3-phosphate dehydrogenase (GAPDH, 1:8000; Kangchen Bio-tech Inc., Shanghai, People's Republic of China). After washing, the membrane was incubated with horseradish-peroxidase-conjugated secondary antibody for 1.5 hours. Immunoreactive bands were detected by a chemiluminescence system (ECL Plus; Amersham, Arlington Heights, IL, USA) and analyzed by Quantity One analysis software. GAPDH was used as the loading control, as in previous reports. ${ }^{30,31}$ Each sample was normalized first against the GAPDH level in the sample, then against the level of the sham sample, as described previously. ${ }^{30,31}$

\section{Immunofluorescence staining}

Mice ( $n=3$ for each group) were anesthetized IP with 4\% chloral hydrate $(0.1 \mathrm{~mL} / 10 \mathrm{~g}$ body weight $)$ and transcardially perfused with $100 \mathrm{~mL}$ of normal saline, followed by $100 \mathrm{~mL}$ of $0.1 \mathrm{M}$ phosphate-buffered saline (PBS) containing 4\% PFA ( $\mathrm{pH}=7.4) .{ }^{28}$ Perfusion-fixed brains were postfixed in $0.1 \mathrm{M}$ PBS $+4 \%$ PFA overnight, followed by dehydration in $40 \%$ sucrose. Coronal brain sections (20 $\mu \mathrm{m}$ thick) were cut on a cryostat (CM1950; Leica Microsystems, Wetzlar, Germany), and the sections were blocked for 1.5 hours in $5 \%$ bovine serum albumin in PBS with $0.1 \%$ Triton $\mathrm{X}-100$. The sections were then incubated overnight at $4{ }^{\circ} \mathrm{C}$ in $3 \%$ bovine serum albumin in $0.1 \%$ Triton $\mathrm{X}-100 / \mathrm{PBS}$ with the primary antibodies: anti-ZO-1 TJ protein antibody (1:50; ab59720) and anti-occludin antibody (1:100; ab31721) (both were purchased from Abcam). After being rinsed three times with PBS, sections were incubated for 2 hours in fluorochromecoupled secondary antibody. The nuclei were stained with 4',6-diamidino-2-phenylindole (DAPI; Boyetime Institute of Biotechnology, Shanghai, People's Republic of China). After being rinsed with PBS, the sections were examined under a fluorescence microscope (Leica Microsystems).

\section{Statistical analysis}

All results are expressed as mean \pm standard deviation. Statistical analysis was carried out using Student's two-tailed $t$-test for comparison between two groups and one-way analysis of variance followed by Dunnett's test when the data involved three or more groups. $P<0.05$ was considered statistically significant. ${ }^{32}$ All analyses were performed with GraphPad Prism software (Version 5.01; GraphPad Software, Inc., La Jolla, CA, USA).

\section{Results}

YQFM reduces infarction volume and brain water content and improves behavioral outcomes in I/R-treated mice

Infarction volume was evaluated in mouse brains by TTC staining and imaging software (Image J software; National Institutes of Health, Bethseda, MD, USA) after I/R injury. 
Representative samples of TTC-stained brain sections are shown in Figure 2A, with corresponding infarction volumes and statistical data that are shown in Figure 2B. Infarct volume was significantly increased in the shamoperated reference group $(37.4 \% \pm 8.6 \%)$, whereas the three doses of YQFM $(0.336,0.671$, and $1.342 \mathrm{~g} / \mathrm{kg})$ reduced the infarct volumes induced by $\mathrm{I} / \mathrm{R}$ by $28.7 \% \pm 3.6 \%$, $25.2 \% \pm 2.7 \%$, and $13.9 \% \pm 1.4 \%$, respectively, with the highest inhibition rate being $62.83 \%$ (YQFM at a dose of $1.342 \mathrm{~g} / \mathrm{kg}$ versus model group). XST at a dose of $40 \mathrm{mg} /$ $\mathrm{kg}$, the positive control, also significantly reduced I/Rinduced infarct volume.

As shown in Figure 2C, there was a marked decline of $10.67 \pm 0.51$ in $\mathrm{I} / \mathrm{R}$ neurological score, while the YQFM and XST treatments resulted in a statistically significant improvement in neurobehavioral deficits compared with the reference group $(P<0.05)$. Furthermore, the reference group had significantly increased brain water content $(80.5 \% \pm 0.6 \%$ versus $78.6 \% \pm 0.7 \%, P<0.05)$, which was significantly attenuated by YQFM at all three doses and by XST (Figure 2D).

\section{YQFM increases CBF in I/R-treated mice}

CBF was determined by a laser Doppler perfusion imaging system in the different groups, as shown in Figure 3A, and the quantification of the results is shown in Figure 3B. The administration of both YQFM at a dose of $1.342 \mathrm{~g} / \mathrm{kg}$ and XST at a dose of $40 \mathrm{mg} / \mathrm{kg}$ resulted in a significant increase in CBF at 24 hours after reperfusion $(63.9 \% \pm 5.9 \%$ versus $27.6 \% \pm 6.4 \%$, YQFM at a dose of $1.342 \mathrm{~g} / \mathrm{kg}$ versus model group, $P<0.05)$, and increased in the other two groups $(0.334$ and $0.671 \mathrm{~g} /$ $\mathrm{kg})$ to a certain extent $(42.1 \% \pm 4.6 \%$ and $51.4 \% \pm 6.0 \%)$. The highest inhibition rate of YQFM was $56.8 \%$ (YQFM at a dose of $1.342 \mathrm{~g} / \mathrm{kg}$ versus model group).

\section{YQFM attenuates BBB disruption in I/R-treated mice}

Quantitative spectrometry detected extensive EB leakage in I/R group compared with the sham group at 24 hours after reperfusion $(2.61 \pm 0.54$ versus $0.21 \pm 0.08 \mu \mathrm{g} / \mathrm{g}, P<0.05)$. YQFM treatment significantly reduced the leakage of EB at all three doses $(1.48 \pm 0.76,1.29 \pm 0.52$, and $0.87 \pm 0.61 \mu \mathrm{g} / \mathrm{g}$ at $0.336,0.671$, and $1.342 \mathrm{~g} / \mathrm{kg}$, respectively), and XST had
A

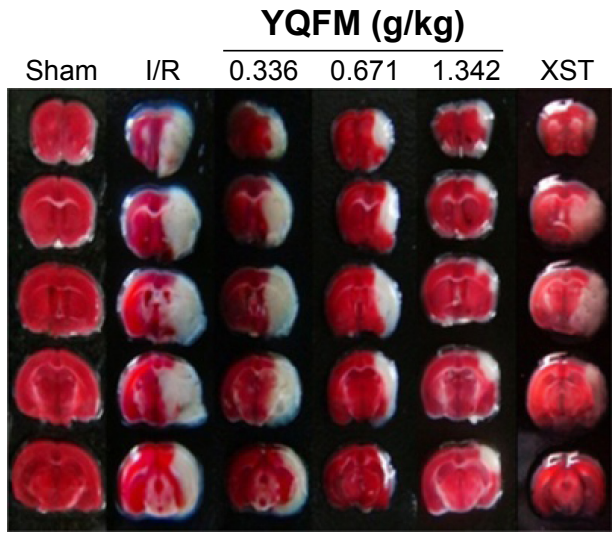

C

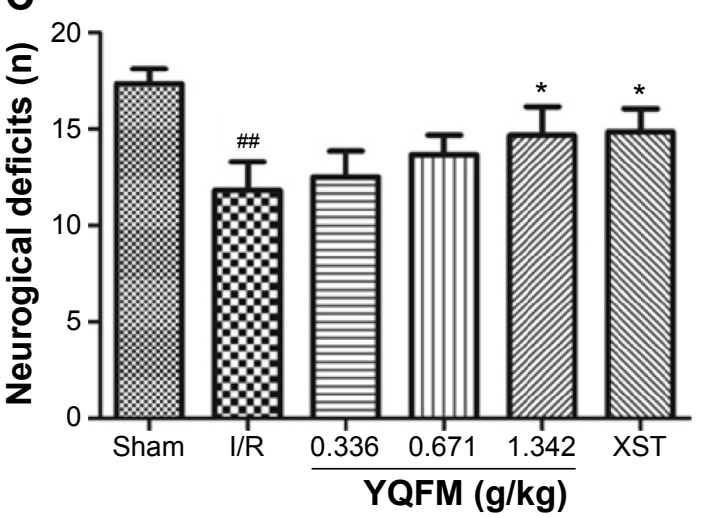

B

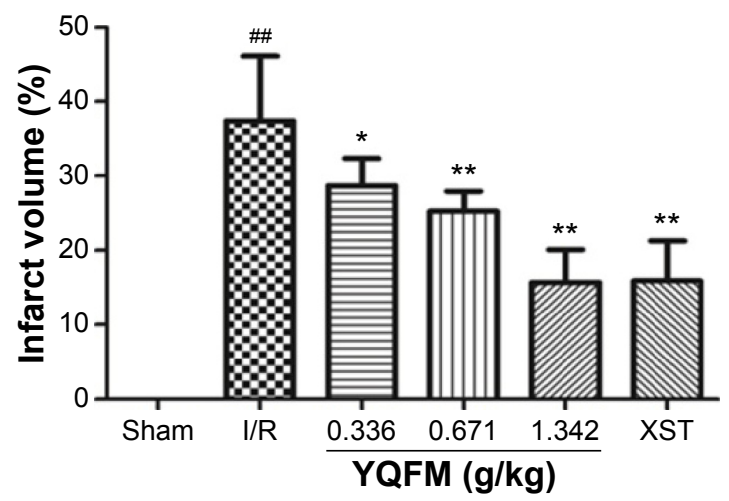

D

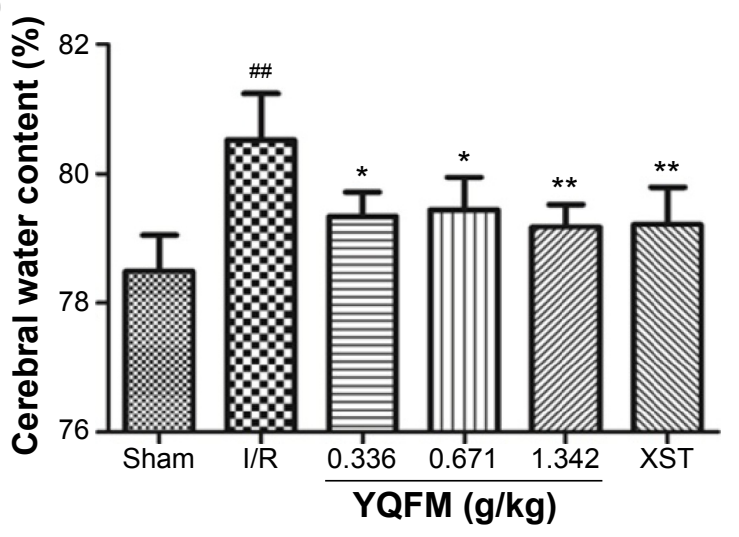

Figure 2 Effects of YQFM on brain injury in mice with cerebral I/R.

Notes: (A, B) infarct volume, (C) neurobehavioral outcomes, and (D) brain water content in mice with cerebral I/R. Mice were subjected to I hour of ischemia and 24 hours of reperfusion. YQFM was administered I hour after ischemia. Data are expressed as mean $\pm S D, n=6$. $P<0.01$ vs sham mice; ${ }^{*} P<0.05$ vs $\mathrm{I} / \mathrm{R}$ mice, ${ }^{* * P}<0.0 \mathrm{I}$ vs I/R mice. Abbreviations: I/R, ischemia-reperfusion; SD, standard deviation; XST, XueShuanTong injection; YQFM, YiQiFuMai powder injection. 

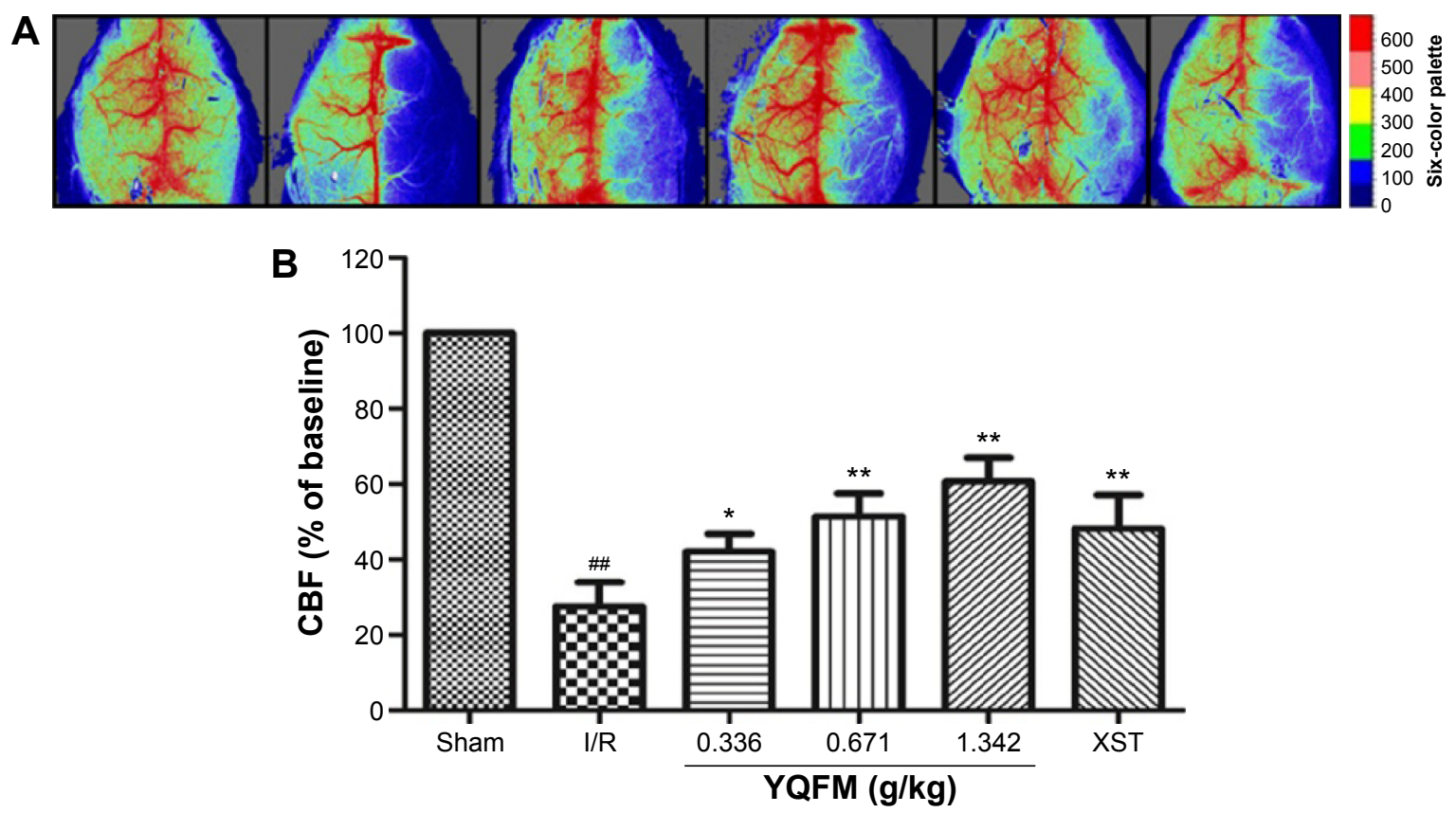

Figure 3 Effect of YQFM on regional cerebral blood flow in mice with cerebral I/R.

Notes: (A) The representative images of cerebral blood flow of ipsilateral cortex in different groups. The magnitude of CBF is represented by different colors, with blue to red denoting low to high. (B) Quantitative analysis of $C B F$ in different groups. Data are expressed as mean $\pm S D, n=6$. ${ }^{\# P} P 0.01$ vs sham mice; $* P<0.05$ vs $I / R$ mice, ${ }^{* *} P<0.01$ vs I/R mice.

Abbreviations: CBF, cerebral blood flow; I/R, ischemia-reperfusion; SD, standard deviation; XST, XueShuanTong injection; YQFM, YiQiFuMai powder injection.

the same effect (Figure 4A and B). The highest inhibition rate of YQFM was $66.67 \%$.

\section{YQFM ameliorates brain metabolism and histopathological damage in I/R-treated mice}

Brain sections of the sham group, the untreated I/R group, and the I/R group treated with YQFM at a dose of $1.342 \mathrm{~g} / \mathrm{kg}$ were examined. Figure $3 \mathrm{~A}$ and $\mathrm{B}$ shows the brain metabolism and histopathological changes after 1 hour of occlusion and 24 hours of reperfusion by micro-PET imaging and H\&E staining, respectively. Brain sections of the I/R group showed weak signal intensity and signal volume in the lesion side (Figure 5A), as well as neuronal loss and the presence of numerous vacuolated spaces (Figure 5B). The data showed that YQFM pretreatment at a dose of $1.342 \mathrm{~g} / \mathrm{kg}$ ameliorated
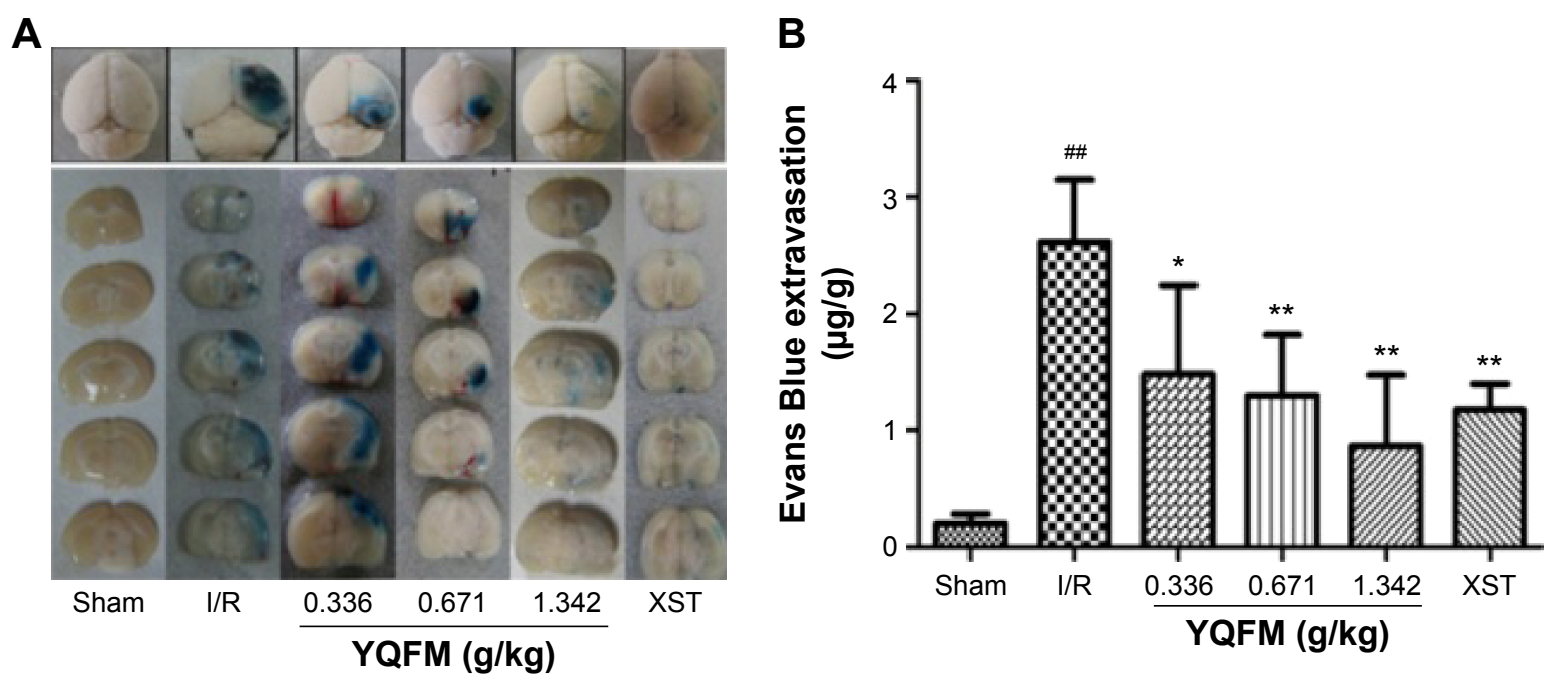

Figure 4 Effect of YQFM on blood-brain barrier permeability in mice with cerebral I/R.

Notes: (A) Representative gross appearance of EB-stained brains from mouse subject to I-hour ischemia followed by 24 hours of reperfusion. (B) Quantitative analysis of EB extravasation by spectrophotometry. Data are expressed as mean $\pm S D, n=6$. $P<0.01$ vs sham mice; $* P<0.05$ vs $l / R$ mice, $* * P<0.01$ vs $l / R$ mice.

Abbreviations: EB, Evans Blue; I/R, ischemia-reperfusion; SD, standard deviation; XST, XueShuanTong injection; YQFM, YiQiFuMai powder injection. 

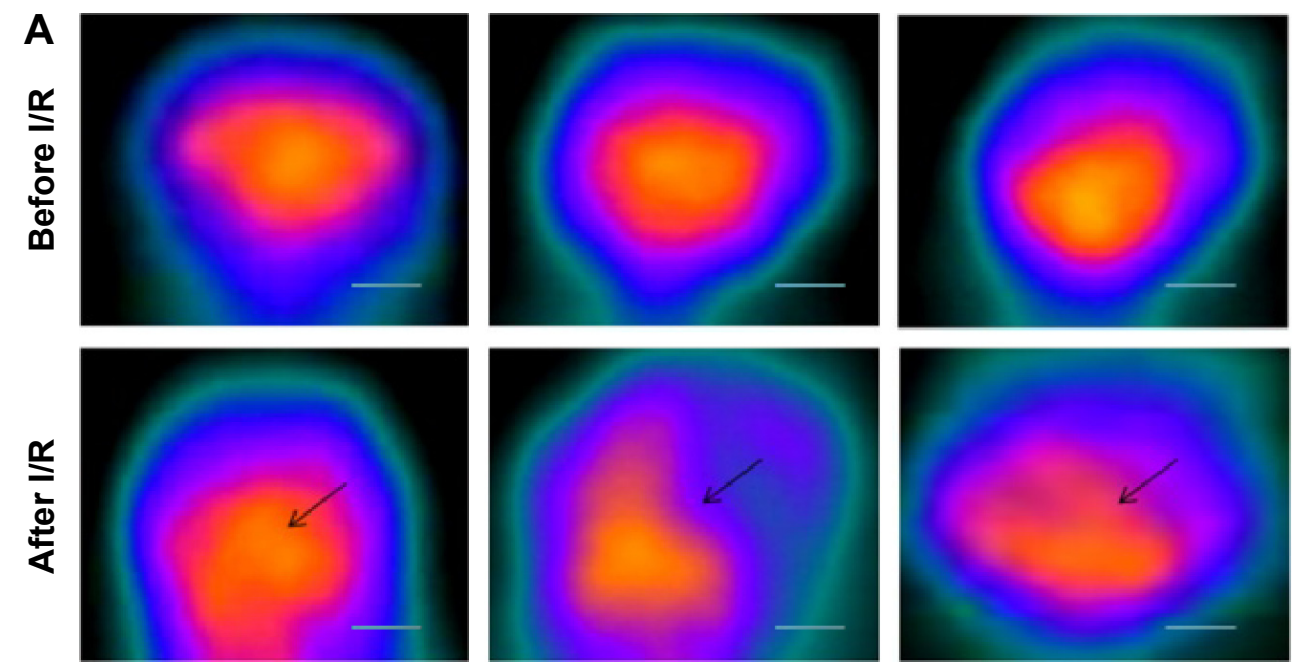

Sham

I/R

YQFM

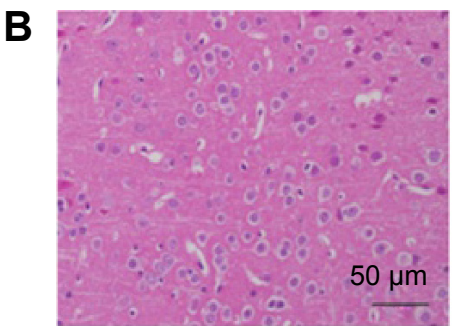

Sham

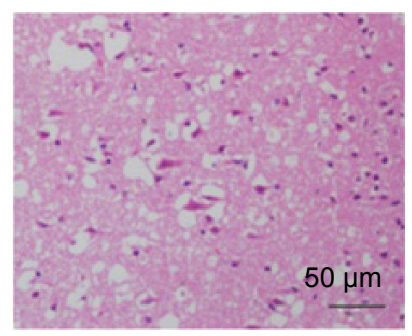

I/R

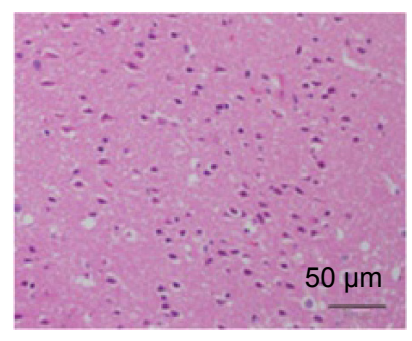

YQFM

Figure 5 Effect of YQFM on histopathological changes of brain sections in mice with cerebral I/R.

Notes: (A) $\left({ }^{18} \mathrm{~F}-\mathrm{FDG}\right)$ PET imaging of a mouse brain before and after $\mathrm{I} / \mathrm{R}$, and representative coronal PET images of $\left({ }^{18} \mathrm{~F}-\mathrm{FDG}\right)$ at the lesion area after surgery. The black arrows indicate the damaged region. Scale bars $=200 \mathrm{~mm}$. (B) Hematoxylin-and-eosin-stained slides of the brain sections of mouse in different groups were examined under a light microscope. Representative stained sections showed a gradual improvement in condensed nuclei in cortical cells in the high-dose YQFM (I.342 g/kg) treatment group. Scale bar $=50 \mu \mathrm{m} ; \mathrm{n}=3$.

Abbreviations: ${ }^{18} \mathrm{~F}-\mathrm{FDG},{ }^{18} \mathrm{~F}-$ fluorodeoxyglucose; I/R, ischemia-reperfusion; PET, positron emission tomography; SD, standard deviation; YQFM, YiQiFuMai powder injection.

brain metabolism and histopathological damage in I/Rtreated mice by increasing the signal intensity and signal volume and decreasing the cell loss.

\section{YQFM increases expression of ZO-I and occludin in I/R-treated mice}

To identify the relationship between $\mathrm{TJ}$ remodeling and BBB integrity, Western blotting and immunofluorescence staining were used to analyze the expression of TJ proteins. Western blot analysis (Figure 6A and B) showed that the expression of ZO-1 and occludin was decreased in the I/R-treated mice, whereas it increased significantly in response to YQFM treatment at a dose of $1.342 \mathrm{~g} / \mathrm{kg}$. No statistical significance was observed with the other two YQFM groups.

We performed CD31/ZO-1 and CD31/occludin double staining, which showed that ZO-1 and occludin were continuously present in the endothelial cell layer of microvessels in normal brains treated with YQFM at a dose of $1.342 \mathrm{~g} / \mathrm{kg}$ (Figure 6C and D). After I/R, ZO-1 and occludin showed structure disruptions with rearrangements compared with sham mice. TJs were much smoother and had more continuous labeling in the group treated with YQFM at a dose of $1.342 \mathrm{~g} / \mathrm{kg}$.

\section{Discussion}

A number of studies have demonstrated that YQFM can be used in cardiovascular diseases with marked efficacy. ${ }^{14,17}$ However, its protective effects in ischemic stroke remain unclear. The results of the current study revealed that YQFM produces a significant improvement in cerebral infarction, neurological score, and brain edema at doses of $0.336,0.671$, and $1.342 \mathrm{~g} / \mathrm{kg}$, showing the same effects as seen with XST (Figure 2A-D). XST, a well-known Chinese herbal preparation, is composed of Panax notoginseng saponins, which shows part similarity to the chemical components of YQFM, and has been approved by the China Food and Drug Administration for the treatment of patients with some ischemic diseases. ${ }^{11,33,34}$ The findings of the current study provide the 
A
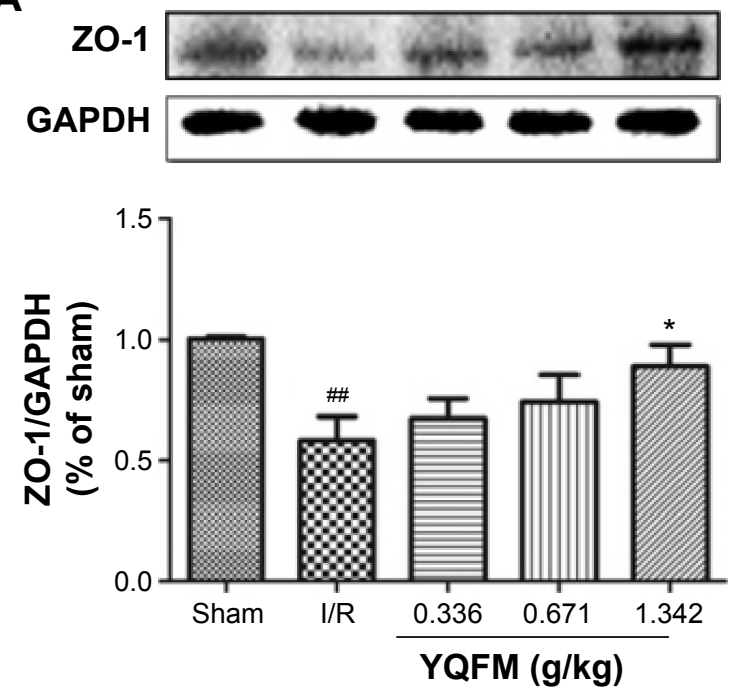

C

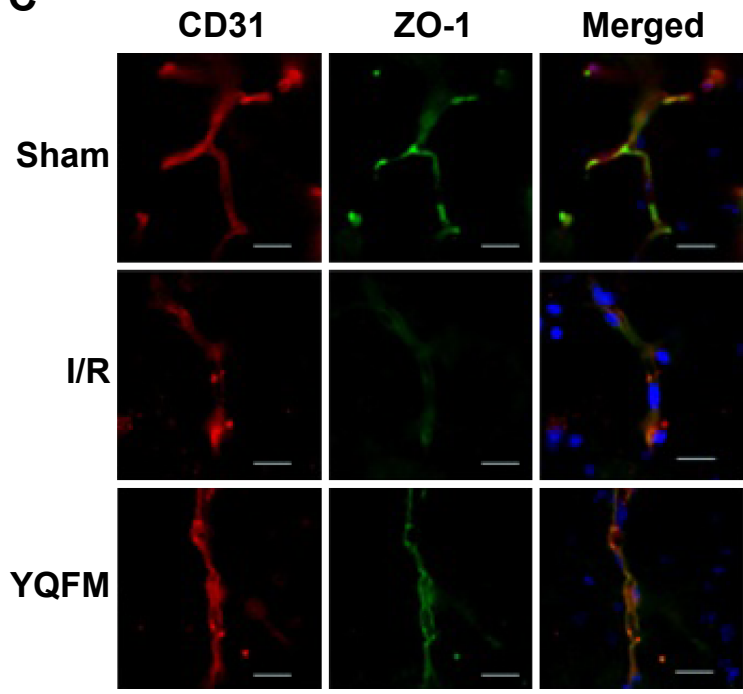

B
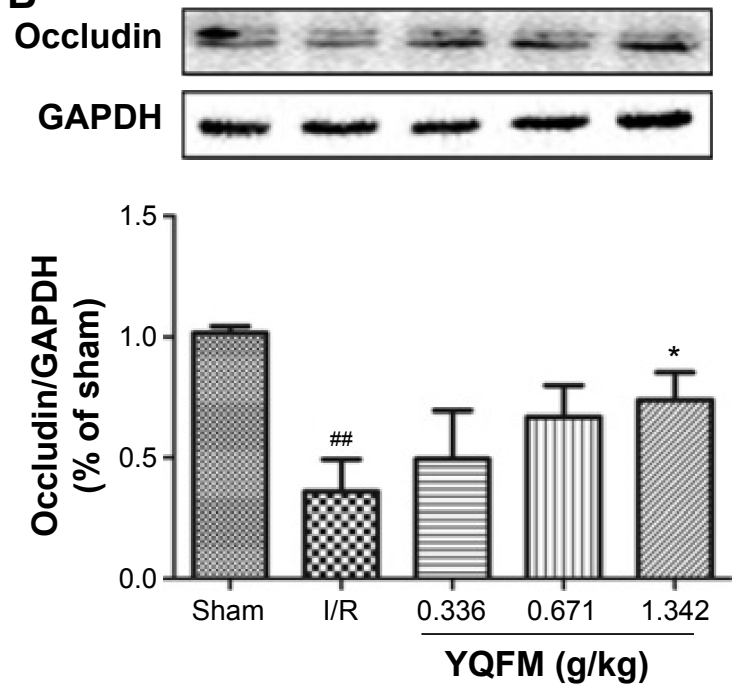

D

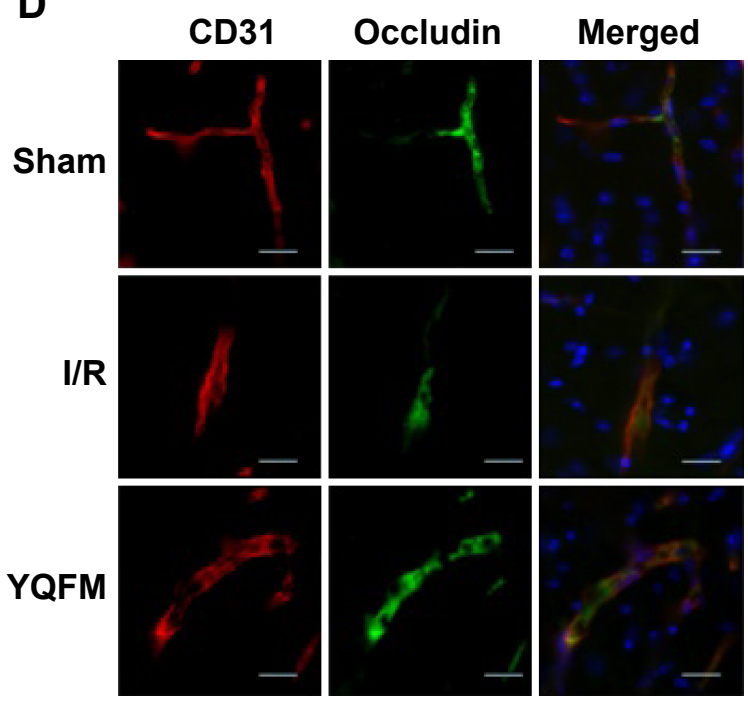

Figure 6 Effect of YQFM on the expression of tight junction proteins in mice with cerebral I/R.

Notes: (A, B) Representative Western blots and the quantitative analysis of the ratio of ZO-I (A) and occludin (B). (C, D) Representative immunofluorescence microscope images of ZO-I (green) and occludin (green) localized at the periphery of endothelial cells with the marker CD3I (red). DAPI-stained nuclei are depicted in blue. Scale bars $=20 \mu \mathrm{m}$. Distribution of ZO-I and occludin was disrupted in the I/R group and was reduced in the high-dose YQFM (I.342 g/kg) treatment group. Data (A, B) are expressed as mean $\pm S D, n=3$. ${ }^{\prime} P<0.0$ I vs sham mice; $* P<0.05$ vs I/R mice.

Abbreviations: GAPDH, glyceraldehyde phosphate dehydrogenase; I/R, ischemia-reperfusion; PET, positron emission tomography; SD, standard deviation; YQFM, YiQiFuMai powder injection; ZO-I, zona occludens-I.

first evidence that YQFM may protect against I/R injury in I/R-treated mice.

Considering that the severity of ischemic damage correlates with the degree of $\mathrm{CBF}$ reduction, ${ }^{35}$ we also investigated the changes in $\mathrm{CBF}$ in the different groups and found that the treatment with YQFM at a dose of $1.342 \mathrm{~g} / \mathrm{kg}$ significantly increased $\mathrm{CBF}$ (Figure $3 \mathrm{~A}$ and $\mathrm{B}$ ), which indicated that the smaller infarct volume in the YQFM-treated groups was correlated with improved CBF during I/R. However, molecular imaging has a number of advantages, such as the visual representation, characterization, and quantification of biological processes at cellular and molecular levels. ${ }^{36,37} \mathrm{PET}$ with ${ }^{18} \mathrm{~F}$-FDG has been widely used in the evaluation of the effects of drugs for cerebrovascular disease via the quantitative and noninvasive monitoring of glucose metabolism. ${ }^{12,38,39}$ In the current study, micro-PET imaging technology was used to further confirm the validity of YQFM treatment in I/R-treated mice. We found that YQFM improved brain glucose metabolism, which was consistent with the results of TTC staining (Figure 5A). In addition, H\&E staining for histopathological observations suggested that neural structure was recovered, with a dense neuropil and largely viable 
neurons, in the brains of the YQFM-treated group compared with the I/R group (Figure 5B). These findings provide further evidence of the effectiveness of YQFM in I/R-treated mice at both the functional and the morphological levels.

Ischemic-stroke-induced brain injury is a complex pathophysiological process, including multifactor, multiway, and multichannel damage. ${ }^{40-43}$ However, BBB disruption is a critical event in the progression of ischemic stroke, which can increase cerebral vascular permeability and lead to the formation of brain edema. ${ }^{44,45}$ Therefore, approaches focusing on the subsequent damage of $\mathrm{BBB}$ dysfunction should be considered. ${ }^{46,47}$ The understanding of BBB dysfunction induced by ischemic stroke following thrombolytic therapy remains a challenge for clinicians. ${ }^{48,49}$ In the current study, the results showed that YQFM treatment significantly reduced the leakage of $\mathrm{EB}$ at three doses (Figure 4A and B), which indicated that YQFM could effectively protect BBB integrity.

Furthermore, it has been generally believed that TJs that mediate paraendothelial transport participate in endothelial cell-mediated BBB permeability. ${ }^{7,50}$ TJs such as ZO-1 and occludin have different molecular structures and characteristics, and their degradation plays a key role in ischemic BBB dysfunction in stroke. ${ }^{51-53}$ Loss and degradation of ZO-1 play significant roles in maintaining the continuity and integrity of TJs, which is closely linked to increases in BBB permeability ${ }^{54}$ Occludin, the first integral transmembrane protein to be identified, is localized to TJs. ${ }^{55}$ Although much has been learned about TJs, the exact mechanisms of action of YQFM on TJs are not clear. Our immunofluorescence microscopy and Western blot assays demonstrated that ZO-1 and occludin participate in the modulation of BBB permeability by YQFM (Figure 6A-D). However, the role of the basal lamina and other TJ proteins in the action of YQFM on BBB permeability is unknown and needs further clarification.

As previously described, ${ }^{18} \mathrm{YQFM}$ consists of bioactive compounds, such as ginsenoside Rb1, ginsenoside Rg1, and schisandrin, some of which display neuroprotective effects after cerebral I/R injury. Recent studies have indicated that ginsenoside $\mathrm{Rb} 1$ can protect the loss of $\mathrm{BBB}$ integrity by suppressing neuroinflammation, while Rg1 attenuates BBB disruption through downregulating the expression of aquaporin 4 induced by ischemic stroke in animals. ${ }^{56,57}$ This indicates that these components identified by UHPLC might be the key active ingredients for BBB protection. Furthermore, the possible mechanisms of action of YQFM are linked with the nuclear factor (NF)- $\mathrm{\kappa B}$ and Akt pathways, ${ }^{58-61}$ and 12 other major signaling pathways, including $\mathrm{NF}-\kappa \mathrm{B}$, mitogen-activated protein kinase, the cytokine network, and mammalian target of rapamycin (mTOR), have been identified as related to the effects of YQFM in cardiocerebral ischemic diseases. ${ }^{19}$ Moreover, ginsenoside Rg1 can ameliorate I/R-induced BBB disruption by regulating protease-activated receptor-1 (PAR-1), while ginsenoside $\mathrm{Rb} 1$ protects $\mathrm{I} / \mathrm{R}$-induced BBB disruption by inhibiting matrix metalloproteinase-9 (MMP-9). ${ }^{56,58}$ These findings indicate that the potential targets or pathways of YQFM in the treatment of ischemic stroke are related to PAR-1, MMP-9, NF- $\kappa$ B, etc. In addition, a combination of four active compounds derived from Sheng-Mai-San was reported to alleviate cerebral I/R injury, correlating with the inhibition of autophagy and modulation of the adenosine monophosphate-activated protein kinase/mTOR and Jun kinase pathways and to inhibit $\mathrm{H}_{2} \mathrm{O}_{2}$-induced $\mathrm{PC} 12$ cell apoptosis in vitro. ${ }^{20,62}$ Ginsenoside $\mathrm{Rg} 1$ can also improve cell viability and cell apoptosis induced by oxygen-glucose deprivation in cultured hippocampal neurons. ${ }^{63}$ These findings suggest that YQFM may attenuate cerebral I/R injury via multiple mechanisms, such as anti-inflammation, antiapoptosis, antioxidation, and inhibition of autophagy, which require further investigation in future studies.

\section{Conclusion}

The current study demonstrates that the compound Chinese medicine YQFM can attenuate I/R-induced brain injury by improving cerebral infarction, neurological score, brain edema, brain metabolism, and histopathological damage. The repair of $\mathrm{BBB}$ disruption is mediated by interference with TJ protein degradation in vascular endothelial cells. These findings provide solid evidence for the efficacy of YQFM in the treatment of ischemic stroke.

\section{Acknowledgments}

This research was supported by funding from the National Natural Science Foundation of the People's Republic of China (grant number 81274004) and the 2011 Program for Excellent Scientific and Technological Innovation Team of Jiangsu Higher Education, a project funded by the Priority Academic Program Development of Jiangsu Higher Education Institutions.

\section{Disclosure}

The authors report no conflicts of interest in this work.

\section{References}

1. Donnan GA, Fisher M, Macleod M, et al. Stroke. Lancet. 2008;371(9624): 1612-1623.

2. Schellinger PD, Kohrmann M. 4.5-hour time window for intravenous thrombolysis with recombinant tissue-type plasminogen activator is established firmly. Stroke. 2014;45(3):912-913. 
3. Fonarow GC, Smith EE, Saver JL, et al. Timeliness of tissue-type plasminogen activator therapy in acute ischemic stroke: patient characteristics, hospital factors, and outcomes associated with door-toneedle times within 60 minutes. Circulation. 2011;123(7):750-758.

4. Adeoye O, Albright KC, Carr BG, et al. Geographic access to acute stroke care in the United States. Stroke. 2014;45(10):3019-3024.

5. Zhang L, Zhang ZG, Chopp M. The neurovascular unit and combination treatment strategies for stroke. Trends Pharmacol Sci. 2012; 33(8):415-422.

6. Lorberboym M, Blankenberg FG, Sadeh M, et al. In vivo imaging of apoptosis in patients with acute stroke: correlation with blood-brain barrier permeability. Brain Res. 2006;1103(1):13-19.

7. Yang Y, Rosenberg GA. Blood-brain barrier breakdown in acute and chronic cerebrovascular disease. Stroke. 2011;42(11):3323-3328.

8. Yilmaz G, Granger DN. Leukocyte recruitment and ischemic brain injury. Neuromolecular Med. 2010;12(2):193-204.

9. Abbott NJ, Patabendige AA, Dolman DE, et al. Structure and function of the blood-brain barrier. Neurobiol Dis. 2010;37(1):13-25.

10. Obermeier B, Daneman R, Ransohoff RM. Development, maintenance and disruption of the blood-brain barrier. Nat Med. 2013;19(12): 1584-1596.

11. Gui Q, Yang Y, Ying S, et al. Xueshuantong improves cerebral blood perfusion in elderly patients with lacunar infarction. Neural Regen Res. 2013;8(9):792-801.

12. Wang Z, Song F, Li J, et al. PET demonstrates functional recovery after treatment by Danhong injection in a rat model of cerebral ischemicreperfusion injury. Evid Based Complement Alternat Med. 2014; 2014:430757.

13. Sun K, Pan CS, Li Q, et al. Ameliorating effects of traditional Chinese medicine on lipopolysaccharide-induced microcirculatory disturbances and organ injury. Endocr Metab Immune Disord Drug Targets. 2015;5(1):8-24.

14. Yuan Q, Wang J, Fang QH, et al. Attenuating effect of pretreatment with Yiqifumai on lipopolysaccharide-induced intestine injury and survival rate in rat. $J$ Inflamm (Lond). 2011;8:10.

15. Yuan Q, Liu YY, Sun K, et al. Improving effect of pretreatment with Yiqifumai on LPS-induced microcirculatory disturbance in rat mesentery. Shock. 2009;32(3):310-316.

16. Li F, Tan Y-S, Chen H-L, et al. Identification of schisandrin as a vascular endothelium protective component in YiQiFuMai powder injection using HUVECs binding and HPLC-DAD-Q-TOF-MS/MS analysis. J Pharmacol Sci. 2015;129:1-8.

17. Xing L, Jiang M, Dong L, et al. Cardioprotective effects of the YiQiFuMai injection and isolated compounds on attenuating chronic heart failure via NF-kappaB inactivation and cytokine suppression. J Ethnopharmacol. 2013;148(1):239-245.

18. Wang YQ, Liu CH, Zhang JQ, et al. Protective effects and active ingredients of yi-qi-fu-mai sterile powder against myocardial oxidative damage in mice. J Pharmacol Sci. 2013;122(1):17-27.

19. Tan Y, Li F, Lv Y, et al. Study on the multi-targets mechanism of YiQiFuMai powder injection on cardio-cerebral ischemic diseases based on network pharmacology. J Proteomics Comput Biol. 2014;1(1):1-9.

20. Guo ZS, Cao GS, Yang HP, et al. A combination of four active compounds alleviates cerebral ischemia-reperfusion injury in correlation with inhibition of autophagy and modulation of AMPK/mTOR and JNK pathways. J Neurosci Res. 2014;92(10):1295-1306.

21. Liu F, McCullough LD. The middle cerebral artery occlusion model of transient focal cerebral ischemia. Methods Mol Biol. 2014;1135: 81-93.

22. Benedek A, Moricz K, Juranyi Z, et al. Use of TTC staining for the evaluation of tissue injury in the early phases of reperfusion after focal cerebral ischemia in rats. Brain Res. 2006;1116(1):159-165.

23. Ahmad A, Genovese T, Impellizzeri D, et al. Reduction of ischemic brain injury by administration of palmitoylethanolamide after transient middle cerebral artery occlusion in rats. Brain Res. 2012;1477:45-58.

24. Mdzinarishvili A, Kiewert C, Kumar V, et al. Bilobalide prevents ischemia-induced edema formation in vitro and in vivo. Neuroscience. 2007;144(1):217-222.
25. Huang $\mathrm{P}$, Zhou CM, Qin H, et al. Cerebralcare Granule(R) attenuates blood-brain barrier disruption after middle cerebral artery occlusion in rats. Exp Neurol. 2012;237(2):453-463.

26. Cen J, Liu L, Li MS, et al. Alteration in P-glycoprotein at the bloodbrain barrier in the early period of MCAO in rats. $J$ Pharm Pharmacol. 2013;65(5):665-672.

27. Endepols H, Mertgens H, Backes H, et al. Longitudinal assessment of infarct progression, brain metabolism and behavior following anterior cerebral artery occlusion in rats. J Neurosci Methods. 2015;253: 279-291.

28. Huang J, Li Y, Tang Y, et al. CXCR4 antagonist AMD3100 protects blood-brain barrier integrity and reduces inflammatory response after focal ischemia in mice. Stroke. 2013;44(1):190-197.

29. Lan R, Xiang J, Wang GH, et al. Xiao-Xu-Ming decoction protects against blood-brain barrier disruption and neurological injury induced by cerebral ischemia and reperfusion in rats. Evid Based Complement Alternat Med. 2013;2013:629782

30. Wang M, Zhong D, Zheng Y, et al. Damage effect of interleukin (IL)-23 on oxygen-glucose-deprived cells of the neurovascular unit via IL-23 receptor. Neuroscience. 2015;289:406-416.

31. Lu YY, Li ZZ, Jiang DS, et al. TRAF1 is a critical regulator of cerebral ischaemia-reperfusion injury and neuronal death. Nat Commun. 2013;4:2852.

32. Soejima Y, Hu Q, Krafft PR, et al. Hyperbaric oxygen preconditioning attenuates hyperglycemia-enhanced hemorrhagic transformation by inhibiting matrix metalloproteinases in focal cerebral ischemia in rats. Exp Neurol. 2013;247:737-743.

33. Gao L, Zhao H, Liu Q, et al. Improvement of hematoma absorption and neurological function in patients with acute intracerebral hemorrhage treated with Xueshuantong. J Neurol Sci. 2012;323(1-2):236-240.

34. Liu H, Liang JP, Li PB, et al. Core bioactive components promoting blood circulation in the traditional Chinese medicine compound xueshuantong capsule $(\mathrm{CXC})$ based on the relevance analysis between chemical HPLC fingerprint and in vivo biological effects. PLoS One. 2014; 9(11):e112675

35. Ma B, Li M, Ma T, et al. Neuroprotective effects of compound FLZ in an ischemic model mediated by improving cerebral blood flow and enhancing Hsp27 expression. Brain Res. Epub 2014 Mar 24.

36. Massoud TF, Gambhir SS. Molecular imaging in living subjects: seeing fundamental biological processes in a new light. Genes Dev. 2003; 17(5):545-580.

37. Cai W. Seeing is believing: molecular imaging in living subjects. Curr Pharm Biotechnol. 2010;11(6):544.

38. Wang J, Chao F, Han F, et al. PET demonstrates functional recovery after transplantation of induced pluripotent stem cells in a rat model of cerebral ischemic injury. J Nucl Med. 2013;54(5):785-792

39. Dong Y, Song F, Ma J, et al. Small-animal PET demonstrates brain metabolic change after using bevacizumab in a rat model of cerebral ischemic injury. Neurosci Bull. 2014;30(5):838-844.

40. Moskowitz MA, Lo EH, Iadecola C. The science of stroke: mechanisms in search of treatments. Neuron. 2010;67(2):181-198.

41. Dutka AJ. A review of the pathophysiology and potential application of experimental therapies for cerebral ischemia to the treatment of cerebral arterial gas embolism. Undersea Biomed Res. 1985;12(4):403-421.

42. Schneider G, Fries P, Wagner-Jochem D, et al. Pathophysiological changes after traumatic brain injury: comparison of two experimental animal models by means of MRI. MAGMA. 2002;14(3):233-241.

43. Luan $\mathrm{H}, \mathrm{Kan} \mathrm{Z}, \mathrm{Xu} \mathrm{Y}$, et al. Rosmarinic acid protects against experimental diabetes with cerebral ischemia: relation to inflammation response. J Neuroinflammation. 2013;10:28.

44. Nanetti L, Raffaelli F, Vignini A, et al. Oxidative stress in ischaemic stroke. Eur J Clin Invest. 2011;41(12):1318-1322.

45. Song J, Cheon SY, Lee WT, et al. The effect of ASK1 on vascular permeability and edema formation in cerebral ischemia. Brain Res. 2015; 1595:143-155.

46. Garbuzova-Davis S, Rodrigues MC, Hernandez-Ontiveros DG, et al. Blood-brain barrier alterations provide evidence of subacute diaschisis in an ischemic stroke rat model. PLoS One. 2013;8(5):e63553. 
47. Zehendner CM, White R, Hedrich J, et al. A neurovascular blood-brain barrier in vitro model. Methods Mol Biol. 2014;1135:403-413.

48. Yepes M, Roussel BD, Ali C, et al. Tissue-type plasminogen activator in the ischemic brain: more than a thrombolytic. Trends Neurosci. 2009; 32(1):48-55.

49. Jin X, Liu J, Yang Y, et al. Spatiotemporal evolution of blood brain barrier damage and tissue infarction within the first $3 \mathrm{~h}$ after ischemia onset. Neurobiol Dis. 2012;48(3):309-316.

50. Wacker BK, Freie AB, Perfater JL, et al. Junctional protein regulation by sphingosine kinase 2 contributes to blood-brain barrier protection in hypoxic preconditioning-induced cerebral ischemic tolerance. $J$ Cereb Blood Flow Metab. 2012;32(6):1014-1023.

51. Krueger M, Bechmann I, Immig K, et al. Blood-brain barrier breakdown involves four distinct stages of vascular damage in various models of experimental focal cerebral ischemia. J Cereb Blood Flow Metab. 2015;35(2):292-303.

52. Li JJ, Xing SH, Zhang J, et al. Decrease of tight junction integrity in the ipsilateral thalamus during the acute stage after focal infarction and ablation of the cerebral cortex in rats. Clin Exp Pharmacol Physiol. 2011;38(11):776-782.

53. Sandoval KE, Witt KA. Blood-brain barrier tight junction permeability and ischemic stroke. Neurobiol Dis. 2008;32(2):200-219.

54. Kago T, Takagi N, Date I, et al. Cerebral ischemia enhances tyrosine phosphorylation of occludin in brain capillaries. Biochem Biophys Res Commun. 2006;339(4):1197-1203.

55. Campbell M, Humphries P. The blood-retina barrier: tight junctions and barrier modulation. Adv Exp Med Biol. 2012;763:70-84.

56. Chen W, Guo Y, Yang W, et al. Protective effect of ginsenoside $\mathrm{Rb} 1$ on integrity of blood-brain barrier following cerebral ischemia. Exp Brain Res. 2015;233(10):2823-2831.
57. Zhou Y, Li HQ, Lu L, et al. Ginsenoside Rg1 provides neuroprotection against blood brain barrier disruption and neurological injury in a rat model of cerebral ischemia/reperfusion through downregulation of aquaporin 4 expression. Phytomedicine. 2014;21(7):998-1003.

58. Xie CL, Li JH, Wang WW, et al. Neuroprotective effect of ginsenoside$\mathrm{Rg} 1$ on cerebral ischemia/reperfusion injury in rats by downregulating protease-activated receptor-1 expression. Life Sci. 2015;121: $145-151$.

59. Zeng XS, Zhou XS, Luo FC, et al. Comparative analysis of the neuroprotective effects of ginsenosides $\mathrm{Rg} 1$ and $\mathrm{Rb} 1$ extracted from Panax notoginseng against cerebral ischemia. Can J Physiol Pharmacol. 2014;92(2):102-108.

60. Lee TH, Jung CH, Lee DH. Neuroprotective effects of Schisandrin B against transient focal cerebral ischemia in Sprague-Dawley rats. Food Chem Toxicol. 2012;50(12):4239-4245.

61. Giridharan VV, Thandavarayan RA, Bhilwade HN, et al. Schisandrin B, attenuates cisplatin-induced oxidative stress, genotoxicity and neurotoxicity through modulating NF-kappa B pathway in mice. Free Radic Res. 2012;46(1):50-60.

62. Shen K, Wang Y, Zhang Y, et al. Cocktail of four active components derived from Sheng-mai San inhibits hydrogen peroxide induced PC12 cell apoptosis linked with caspase-3/ROCK1/MLC pathway. Rejuvenation Res. Epub 2015 Sep 29.

63. He Q, Sun JG, Wang Q, et al. Neuroprotective effects of ginsenoside Rg1 against oxygen-glucose deprivation in cultured hippocampal neurons. J Chin Med Assoc. 2014;77(3):142-149.
Drug Design, Development and Therapy

\section{Publish your work in this journal}

Drug Design, Development and Therapy is an international, peerreviewed open-access journal that spans the spectrum of drug design and development through to clinical applications. Clinical outcomes, patient safety, and programs for the development and effective, safe, and sustained use of medicines are a feature of the journal, which

\section{Dovepress}

has also been accepted for indexing on PubMed Central. The manuscript management system is completely online and includes a very quick and fair peer-review system, which is all easy to use. Visit http://www.dovepress.com/testimonials.php to read real quotes from published authors.

Submit your manuscript here: http://www.dovepress.com/drug-design-development-and-therapy-journal 